\title{
Determining the GRB (Redshift, Luminosity)-Distribution Using Burst Variability
}

\author{
Timothy Donaghy*, Donald Q. Lamb*, Daniel E. Reichart ${ }^{\dagger}$ and Carlo Graziani* \\ ${ }^{*}$ Department of Astronomy \& Astrophysics, University of Chicago, 5640 South Ellis Avenue, Chicago, IL 60637 \\ ${ }^{\dagger}$ Department of Astronomy, California Institute of Technology, 1201 East California Boulevard, MS 105-24, \\ Pasadena, CA 91125
}

\begin{abstract}
We use the possible Cepheid-like luminosity estimator for the long-duration gamma-ray bursts (GRBs) developed by Reichart et al. (2000) to estimate the intrinsic luminosity, and thus the redshift, of 907 long-duration GRBs from the BATSE 4B catalog. We describe a method based on Bayesian inference which allows us to infer the intrinsic GRB burst rate as a function of redshift for bursts with estimated intrinsic luminosities and redshifts. We apply this method to the above sample of long-duration GRBs, and present some preliminary results.
\end{abstract}

\section{INTRODUCTION}

There is increasing evidence that gamma-ray bursts (GRBs) are due to the collapse of massive stars (see, e.g., [1] for a discussion of this evidence). If GRBs are indeed related to the collapse of massive stars, one expects the GRB rate to be roughly proportional to the star-formation rate (SFR). However, the observed redshift distribution of GRBs differs noticeably from that of the SFR: the observed GRB redshift distribution peaks at $z \approx 1$ and few bursts are observed beyond $z \sim 1.5$, while the SFR peaks at $z \approx 2$ and $10-40 \%$ of stars are thought to form beyond $z=5$ (see, e.g., [2,3]).

However, observational selection effects play a key role in determining the observed redshift distribution of GRBs. Among these selection effects are the efficiencies with which burst redshifts can be determined by spectroscopic observations of the burst afterglow and/or the host galaxy of the burst. In addition, both of these methods of determining redshift require the identification of an optical afterglow of the GRB, and the detectability of the optical afterglow may be a function of redshift.

In this paper, we take a different approach. We use the possible Cepheid-like luminosity estimator for the long-duration GRBs developed by Reichart et al. [4] to estimate the intrinsic luminosity, and thus the redshift, of 907 long-duration GRBs from the BATSE 4B catalog [5]. This approach is free of the important and difficult-to-quantify observational selection effects that affect the redshift distribution of the GRBs with known redshifts. We assume a very general model for the GRB rate and a truncated power-law model for the intrin-

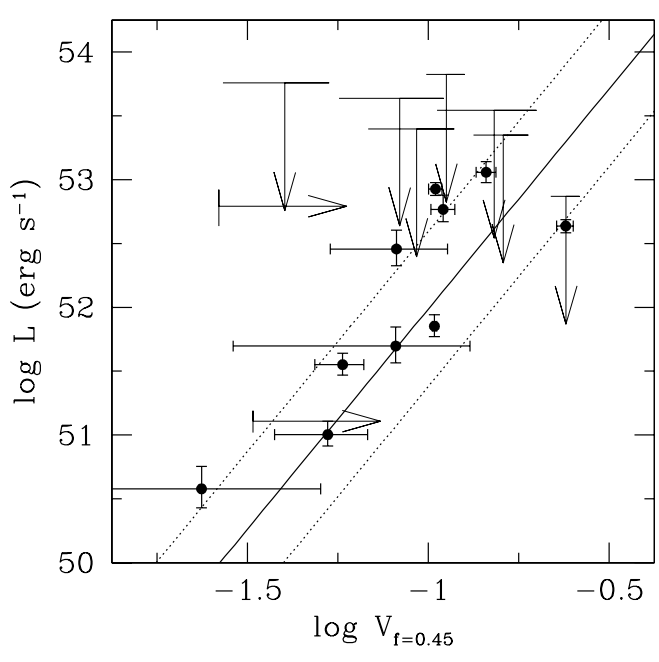

FIGURE 1. The variabilities $V$ and isotropic-equivalent peak photon energy luminosities $L$ of the 19 bursts for which some redshift information exists. The solid and dotted lines mare the center and 1- $\sigma$ widths of the best-fit model of these bursts in the $(\log L, \log V)$-plane.

sic GRB isotropic-equivalent photon luminosity distribution (in this work we assume that the amplitude and the power-law index of the isotropic-equivalent photon luminosity distribution does not evolve; we will relax this assumption in future work). Adopting a Bayesian approach, we calculate the likelihood of the data given the model, and convert it to a posterior distribution on the model parameters. 
GRB Catalog

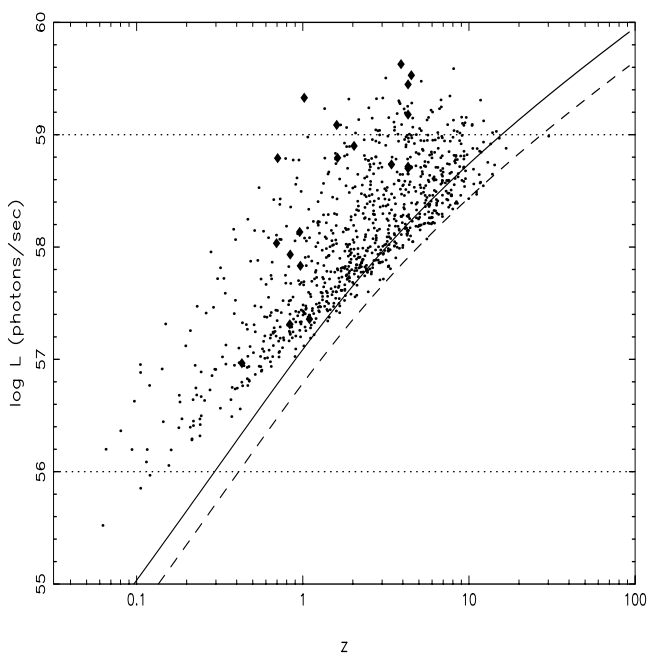

Simulated GRB Catalog

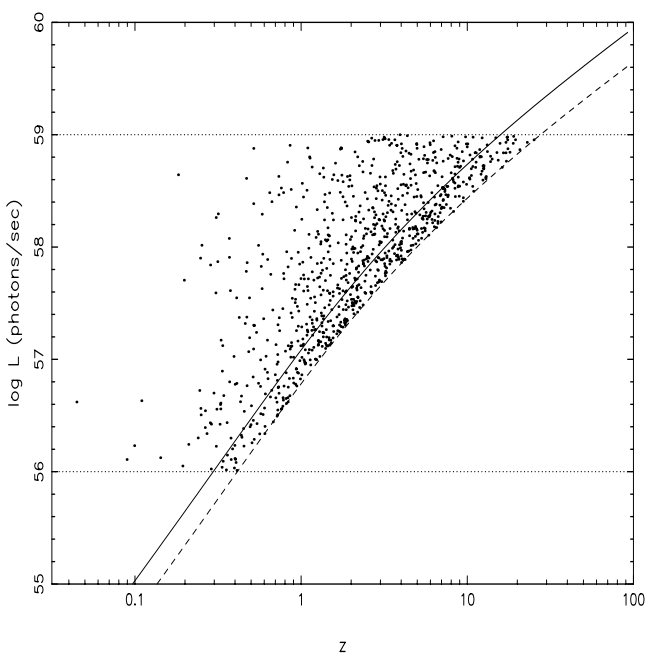

FIGURE 2. Left panel: distribution of the 907 long-duration BATSE bursts in the $(\log z, \log L)$-plane, as determined by our luminosity estimator. The solid and dashed curves represent the $90 \%$ and $10 \%$ detection thresholds of BATSE. The horizontal dotted lines show the values of $L_{\mathrm{min}}$ and $L_{\mathrm{max}}$ adopted in this study. The diamonds show the locations of the 19 GRBs with known redshifts used to calibrate the luminosity estimator. Right panel: a Monte Carlo simulation of the expected distribution of $\approx 900$ bursts with peak photon fluxes $P$ above the BATSE $90 \%$ detection threshold, assuming the maximum likelihood best-fit parameters for the model (see text).

\section{LUMINOSITY ESTIMATOR}

A possible Cepheid-like luminosity estimator for gamma-ray bursts has been suggested by Ramirez-Ruiz and Fenimore [6] and developed further by Reichart et al. $[4,7,8]$. These authors have shown that there exists a correlation between a measure $V$ of the variability of the burst time history and the intrinsic isotropic-equivalent peak photon energy luminosity $L$ of the burst for the 19 GRBs for which some redshift information (either a redshift measurement or a redshift limit) exists. We show this correlation in Figure 1.

We apply this luminosity estimator to 907 bursts from the BATSE 4B catalog [5] that have durations $T_{90}>10$ sec. We show the resulting redshift-luminosity distribution for these bursts in Figure 2 (see also [7] and [8]).

\section{MODEL}

In this work, we assume that the rate of GRBs per unit redshift and luminosity is given by a separable function of redshift $z$ and intrinsic isotropic-equivalent photon number luminosity $L$. That is, we assume that the intrinsic photon number luminosity distribution of GRBs does not evolve with redshift; we will relax this assumption in future work. Then the rate of GRBs per unit redshift and luminosity can be written as [9]

$$
\frac{d N}{d z d L_{N}}=\rho(z) f(L) \text {, }
$$

where

$$
\rho(z)=R_{\mathrm{GRB}}(z ; P, Q) \times(1+z)^{-1} \times 4 \pi r(z)^{2}(d r / d z)
$$

is the rate of GRBs that occur at redshift $z$, and $r(z)$ is the comoving distance to the source.

We adopt a phenomenological model for the rate of GRBs that occur at redshift $z$ per unit comoving volume of the form proposed by Rowan-Robinson for the star formation rate [10]. In this model, the GRB rate is given by

$$
R_{\mathrm{GRB}}(z ; P, Q)=R_{0}\left(\frac{t(z)}{t(0)}\right)^{P} \exp \left[-Q\left(1-\frac{t(z)}{t(0)}\right)\right]
$$

where $P, Q$ and $R_{0}$ are model parameters, and $t(z)$ is the time since the Big Bang corresponding to the redshift $z$.

We take the intrinsic photon luminosity distribution of GRBs to be a truncated power-law,

$$
\begin{aligned}
f(L)= & \frac{1-\beta}{L_{\max }^{1-\beta}-L_{\min }^{1-\beta}} L^{-\beta} \\
& \times \Theta\left(L-L_{\min }\right) \Theta\left(L_{\max }-L\right) .
\end{aligned}
$$

Thus the model has six parameters: $P, Q, R_{0}, \beta, L_{\text {min }}$, and $L_{\text {max }}$.

In this work we assume a flat universe with $\Omega_{M}=0.3$ and $\Omega_{\Lambda}=0.7$. Furthermore, we fix $L_{\min }$, and $L_{\max }$ to be constants (see Figure 2), reducing the number of free parameters in our model to four. 

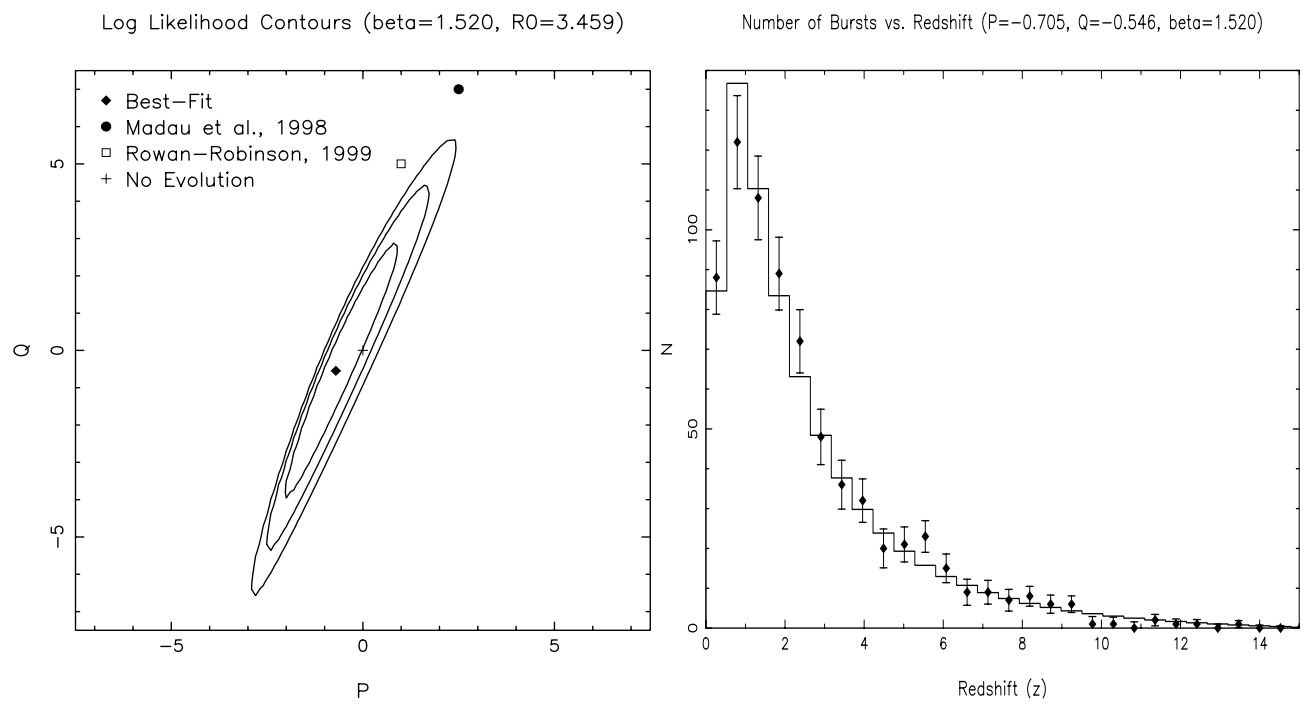

FIGURE 3. Left panel: the best-fit point in the $(P, Q)$-plane at the maximum likelihood best-fit values of the normalization $R_{0}=3.46$ of the GRB rate and the power-law index $\beta=1.52$ of the GRB luminosity distribution. The contours shown correspond to $\Delta \log \mathcal{L}=150$. Right panel: a comparison of the expected and observed number of bursts with $P$ above the BATSE $90 \%$ detection threshold for the maximum likelihood best-fit model.

\section{LIKELIHOOD FUNCTION}

The likelihood function for GRBs that are Poisson in time is given by [9]

$$
\mathcal{L}=\exp \left\{-\int d z d P \mu(z, P) \varepsilon(z, P)\right\} \prod_{i=1}^{N} \mu\left(z_{i}, P_{i}\right),
$$

where the event likelihood,

$$
\begin{aligned}
\mu\left(z_{i}, P_{i}\right)= & \int_{0}^{\infty} d L \rho\left(z_{i}\right) f(L) \delta\left(P_{i}-\frac{L}{4 \pi r\left(z_{i}\right)^{2}\left(1+z_{i}\right)^{\alpha}}\right) \\
= & \rho\left(z_{i}\right) \times f\left(4 \pi r\left(z_{i}\right)^{2}\left(1+z_{i}\right)^{\alpha} P_{i}\right) \\
& \times 4 \pi r\left(z_{i}\right)^{2}\left(1+z_{i}\right)^{\alpha}
\end{aligned}
$$

is the expected number of events observed within $d z d P$ of redshift $z_{i}$ and peak photon number flux $P_{i}$. The quantity $\alpha$ is the burst spectral index, which we take to be equal to 1.0, a value that is typical of GRBs [11]. By an application of Bayes' Theorem, we regard $\mathcal{L}$ as an (unnormalized) probability distribution on the model parameters. We estimate the best-fit parameters in our model by maximizing this likelihood function over the parameter space.

We take the BATSE observing efficiency $\varepsilon(z, P)$, which appears in the exponential factor in the likelihood function, to be $\theta\left(P-P_{t h}\right)$, where $P_{t h}=0.4$ photons $\mathrm{cm}^{-2}$ $\mathrm{sec}^{-1}$ is the $90 \%$ BATSE detection threshold. The product of the event likelihoods $\mu\left(z_{i}, P_{i}\right)$ runs over the 907 BATSE bursts considered in this study.

\section{RESULTS}

Each $(z, L)$-point in the left panel of Figure 2 corresponds to the maximum value of a probability distribution for that particular burst. Thus each point has statistical and systematic errors that are associated with it and that are not displayed in the figure. Since the estimate of the redshift $z$ is calculated from the estimate of the peak photon luminosity $L$ (and the peak observed photon number flux $P$ ), the errors in $z$ and $L$ are completely correlated; that is, the uncertainty lies along curves of constant $P$ (i.e., they are diagonals) in the $(z, L)$-plane. Furthermore, these uncertainties are not symmetric; they are skewed toward low $L$ at low $z$ and toward high $L$ at high $z[7,8]$.

In this preliminary work, we neglect these statistical and systematic errors, and use only the best-fit values in our analysis. Calculating the likelihood of this data, given the model, and converting it to a posterior distribution on the model parameters, we find the maximum likelihood parameters for this data set to be: $P=-0.70, Q=-0.55$, $R_{0}=3.46$ and $\beta=1.52$. The right panel of Figure 2 shows a Monte Carlo simulation of the distribution of $\approx 900$ bursts whose peak photon fluxes $P$ are above the BATSE $90 \%$ detection threshold for the maximum likelihood best-fit parameters.

The left panel of Figure 3 shows the best-fit point and contours of $\Delta \log \mathcal{L}=150$ in the $(P, Q)$-plane at the maximum likelihood best-fit values of the normalization $R_{0}=3.46$ of the GRB rate and the power-law in$\operatorname{dex} \beta=1.52$ of the GRB luminosity distribution. The left panel of Figure 3 thus represents a two-dimensional slice through the four-dimensional parameter space of 

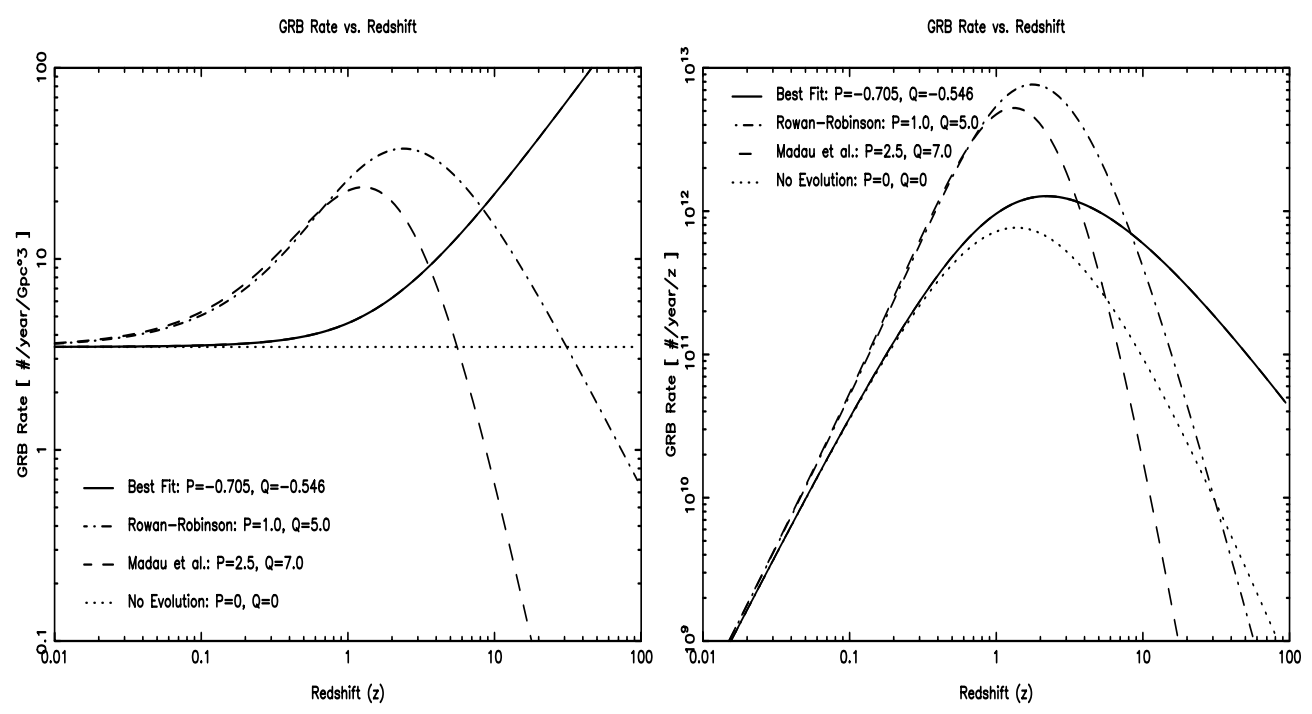

FIGURE 4. Left panel: the best-fit GRB rate per unit comoving volume (solid line). Right panel: the GRB rate per unit redshift $z$. Estimates of the star formation rate as a function of redshift $z$ made by Madau et al. (1998) and Rowan-Robinson (1999) are shown in both panels for comparison, as is the no evolution with redshift $z$ model $(P=Q=0)$. We emphasize that we have not taken into account the statistical and systematic errors in the redshifts $z$ and intrinsic peak photon luminosities $L$ derived from the variability measure $V$, and therefore cannot quote meaningful confidence regions for our best-fit parameters.

the model. The contours shown in Figure 3 do not correspond to credible regions because we have not taken into account the statistical and systematic errors in the redshifts $z$ and intrinsic peak photon luminosities $L$ derived from the variability measure $V$. However, taking the preliminary results at face value, the best-fit model appears to be consistent with no evolution but to be inconsistent with the Rowan-Robinson [10] and the Madau et al. [12] expressions for the star formation rate as a function of $z$.

The right panel of Figure 3 shows a comparison of the observed and expected numbers of bursts with $P$ above the BATSE $90 \%$ detection threshold for the maximum likelihood best-fit model. These distributions thus correspond to projections onto the $z$-axis of the burst distributions in the left and right panels of Figure 2, respectively.

The left panel of Figure 4 shows the best-fit GRB rate per unit comoving volume, while the right panel shows the GRB rate per unit $z$. Estimates of the star formation rate as a function of $z$ made by Madau et al. (1998) and Rowan-Robinson (1999) are shown for comparison, as is the no evolution model $(P=Q=0)$.

Applying the intrinsic peak luminosity estimator of Ramirez-Ruiz and Fenimore [5] to the brightest 220 long-duration bursts in the BATSE 4B catalog [11], Lloyd-Ronning et al. [13] find that the GRB rate increases with increasing redshift $\left(R_{\mathrm{GRB}} \propto(1+z)^{3}\right.$ until $z \sim 2$ and $\propto(1+z)^{1}$ from $z \sim 2$ until $\left.z \sim 10\right)$, and that the mean luminosity of the GRBs also increases with increasing redshift $\left(<L>\propto(1+z)^{1.4 \pm 0.2}\right)$. The results found in the present preliminary work differ somewhat from these conclusions. But we again emphasize that (1) the model that we have used in the present preliminary study does not allow for the evolution of $L$ with $z$, and (2) we have not taken into account the statistical and systematic errors in the redshifts $z$ and intrinsic peak photon luminosities $L$ derived from the variability measure $V$, and therefore cannot quote meaningful confidence regions for our best-fit parameters. In future work, we will relax the assumption of no evolution and will take into account the statistical and systematic errors.

\section{REFERENCES}

1. Lamb, D. Q. 2000, Phys. Reports, 333, 505

2. Gnedin, N. Y., \& Ostriker, J. P. 1997, ApJ, 486, 581

3. Valageas, P., \& Silk, J. 1999, A\&A, 347, 1

4. Reichart, D., et al. 2001 ApJ, 552, 57

5. Paciesas, W. S. et al. 1999, ApJS, 122, 465

6. Ramirez-Ruiz, E. \& Fenimore, E. E. 2000, ApJ, submitted (astro-ph/0004176)

7. Reichart, D. E. and Lamb, D. Q. 2001a, in Gamma-Ray Bursts in the Afterglow Era, ed. E. Costa, F. Frontera, and J. Hjorth (Springer-Verlag; Berlin), 233

8. Reichart, D. E. and Lamb, D. Q. 2001b, in Relativistic Astrophysics, AIP Conference Proceedings No. 586, ed. J. C. Wheeler and H. Martel (AIP: New York), p. 599

9. Weinberg, N., Graziani, C., Lamb, D. \& Reichart, D., 2001, in Gamma-Ray Bursts in the Afterglow Era, ed. E. Costa, F. Frontera, and J. Hjorth (Springer: Berlin), 252

10. Rowan-Robinson, M., 1999, Ap\&SS, 266, 291

11. Mallozzi, R. S., et al. 1996, ApJ, 471, 636

12. Madau, P., et al. 1998, ApJ, 498, 106

13. Lloyd-Ronning, N. M. et al. (astro-ph/0108200) 